\title{
Virulence characteristics of multidrug resistant biofilm forming Acinetobacter baumannii isolated from intensive care unit patients
}

\author{
Habib Zeighami ${ }^{1}$, Fatemeh Valadkhani ${ }^{1}$, Reza Shapouri ${ }^{2}$, Elham Samadi ${ }^{1}$ and Fakhri Haghi ${ }^{1 *}$
}

\begin{abstract}
Background: Nosocomial infections and persistence of multidrug resistant biofilm forming Acinetobacter baumannii in hospitals has made it as a serious problem in healthcare settings worldwide.

Methods: A total of 100 A. baumannii clinical isolates from immunocompromised patients hospitalized in ICU were investigated for biofilm formation, the presence of biofilm related genes (bap, ompA, csuE, fimH, epsA, bla $a_{P E R-1,}$ bfmS, ptk, pgaB, csgA, kpsMII), integron characterization and molecular typing based on REP-PCR.

Results: All isolates were resistant to three or more categories of antibiotics and considered as multidrug resistant (MDR). A total of 32 isolates were resistant to all tested antibiotics and 91\% were extensively drug-resistance (XDR). All isolates were able to produce biofilm and $58 \%$ of isolates showed strong ability to biofilm formation. All strong biofilm forming A. baumannii isolates were XDR. All A. baumannii isolates carried at least one biofilm related gene. The most prevalent gene was csuE (100\%), followed by pgaB (98\%), epsA and ptk (95\%), bfms (92\%) and ompA (81\%). $98 \%$ of isolates carried more than 4 biofilm related genes, simultaneously. Class I integron (67\%) was more frequent in comparison with class II (10\%) $(P<0.05)$. The REP-PCR patterns were classified as 8 types $(\mathrm{A}-\mathrm{H})$ and 21 subtypes. The A1 (23\%) and C1 (15\%) clusters were the most prevalent among $A$. baumannii isolates $(P<0.05)$. According to the REP-PCR patterns, $23 \%$ of all isolates had a clonal relatedness.

Conclusion: Our study revealed the high frequency of biofilm forming XDR A. baumannii in ICU patients, with a high prevalence of biofilm related genes of csuE and pgaB. It seems that the appropriate surveillance and control measures are essential to prevent the emergence and transmission of XDR A. baumannii in our country.
\end{abstract}

Keywords: Acinetobacter baumannii, Biofilm, Virulence factor, Integron, REP-PCR

\section{Background}

Acinetobacter baumannii is an important opportunistic human pathogen that causes a variety of infections as ventilator-associated pneumonia, meningitis, bacteremia, wound and soft-tissue infections, peritonitis and urinary tract infections [1, 2]. High prevalence of multidrug resistant (MDR) A. baumannii has emerged as serious problem in healthcare settings in Iran [2, 3]. Persistence and survival ability of MDR A. baumannii in various

\footnotetext{
*Correspondence: haghi@zums.ac.ir

${ }^{1}$ Department of Microbiology, School of Medicine, Zanjan University of Medical Sciences, Zanjan, Iran

Full list of author information is available at the end of the article
}

hospital environments and dry condition has made it as a major cause of nosocomial infections worldwide [4]. One of the important factors contributing in chronic and persistence infections and antimicrobial resistance of A. baumannii is its capability to colonize and form biofilm on biotic and abiotic surfaces [5]. The biofilm formation rate in A. baumannii is $80 \sim 91 \%$ which is higher than other species (5 24\%) [6]. Previous studies have reported a positive relationship between biofilm formation and antibiotic resistance in A. baumannii isolates [7]. Several virulence factors involved in bioflm formation of $A$. baumannii such as the outer membrane protein A (OmpA), biofilm associated protein (Bap), 
chaperon-usher pilus (Csu), extracellular exopolysaccharide (EPS), two-component system (BfmS/BfmR), poly- $\beta-(1,6)-\mathrm{N}$-acetyl glucosamine (PNAG) and quorum sensing system $[4,5]$.

Biofilm associated protein (Bap) is a large cell surface protein (854-kDa) and homologous to Staphylococcal Bap protein that plays a critical role in cell to cell interactions and biofilm maturation $[4,8]$. The $38-\mathrm{kDa}$ outer membrane protein OmpA as major porin of A. baumannii plays an important role in attachment and invasion to epithelial cells via interaction with fibronectin. This protein is also involved in serum resistance, biofilm formation and persistence, induction of apoptosis and antimicrobial resistance of A. baumannii $[1,4,8]$. Furthermore, previous studies have shown that biofilm formation and attachment of A. baumannii to respiratory epithelial cells enhanced in the presence and expression of betalactamase $b l a_{P E R-1}$ gene [5]. The CsuA/BABCDE chaperone-usher pilus is necessary for the initiation of biofilm formation on abiotic surfaces. It has been shown that inactivation of the $c s u E$ gene eliminates pilus production and biofilm formation $[4,9]$. The expression of csu operon is regulated by a two-component system, bfmRS. The bfmRS system consists of bfmS as a histidine sensor kinase gene which senses environmental conditions and $b f m R$ as response regulator encoding gene. According to previous reports, inactivated $b f \mathrm{mS}$ reduce bioflm formation in A. baumannii type strain 17978 [5, 9]. The extracellular polysaccharide poly- $\beta-(1,6)-\mathrm{N}$-acetyl glucosamine (PNAG) is also involved in biofilm formation, virulence, immune evasion and antibiotic resistance [9].

The present study aimed to investigate the biofilm related genes (bap, ompA, csuE, fimH, epsA, bla PER-1 $_{1}$, bfmS, ptk, pgaB, csgA, kpsMII), integron characterization and molecular typing based on REP-PCR in multidrug resistant $A$. baumannii isolated from immunocompromised patients hospitalized in ICU.

\section{Methods}

\section{Bacterial isolates}

In our previous study, a total of 100 non-replicate $A$. baumannii clinical isolates were randomly recovered from different specimens including blood, sputum, wound swabs, chest tube secretions and urine from immunocompromised patients with symptomatic clinical infections at least $48 \mathrm{~h}$ after ICU admission [2]. Case patients were defined as patients infected by $A$. baumannii according to the Centers for Disease Control and Prevention criteria. The patients who were colonized with A. baumannii and immunocompetent patients were excluded. Informed consent and ethical approval was obtained from management of the hospitals prior to the study. The isolates were identified as A. baumannii using biochemical tests and PCR targeting the $b a_{O X A-51}$ gene.

\section{Antimicrobial susceptibility testing}

The antibiotic susceptibility was determined using the disk diffusion method in our previous study [2]. The following antimicrobial disks were used: ampicillin-sulbactam (10/ $10 \mu g)$, ceftazidim $(30 \mu \mathrm{g})$, imipenem $(10 \mu \mathrm{g})$, gentamicin $(10 \mu \mathrm{g})$, tobramycin $(10 \mu \mathrm{g})$, doxycycline $(30 \mu \mathrm{g})$, ciprofloxacin $(5 \mu \mathrm{g})$, levofloxacin $(5 \mu \mathrm{g})$, co-trimoxazole (1.25/ $23.75 \mu \mathrm{g})$, piperacillin $(100 \mu \mathrm{g})$ and cefepime $(30 \mu \mathrm{g})$ (MAST, Merseyside, U.K). The results were interpreted according to the CLSI guidelines [10]. Multidrug resistance (MDR) was defined as resistance to at least one agent in three or more categories of antibiotics. A. baumannii isolates with resistance to at least one agent in all but two or fewer antimicrobial categories was considered as extensively drug-resistance (XDR) [11].

\section{Biofilm formation}

Biofilm-forming capacity of $A$. baumannii isolates was determined using a microtitre plate assay as described previously [12]. A. baumannii isolates were grown overnight in trypticase soy broth (TSB) containing $0.25 \%$ glucose at $37^{\circ} \mathrm{C}$. Free cells were removed and biofilms were washed three times with sterile phosphate-buffered saline (PBS) and fixed with $150 \mathrm{~mL}$ of $99 \%(\mathrm{v} / \mathrm{v})$ methanol (Merck). The wells were stained with $1 \%(\mathrm{w} / \mathrm{v})$ crystal violet for $20 \mathrm{~min}$ at room temperature. Crystal violet was dissolved using $33 \%(\mathrm{v} / \mathrm{v})$ ethanol/acetone $(80,20, \mathrm{v} /$ v) for $20 \mathrm{~min}$ and the absorbance was measured at 595 nm. Biofilm formation was scored as follows: , nonbiofilm forming (A595 < 1); +, weak $(1<$ A595 $\leq 2)$; ++, moderate $(2<\mathrm{A} 595 \leq 3) ;+++$, strong (A595 >3). Reported values are the mean of three measurements.

\section{DNA extraction}

Genomic DNA was extracted from overnight culture of A. baumannii isolates using a QIAGEN DNA Mini kit (QIAGEN Inc., Valencia, CA).

\section{Detection of biofilm related genes}

The presence of the biofilm related genes bap, ompA, csuE, fimH, eps $A, b l a_{P E R-1}, b f m S, p t k, p g a B, \operatorname{csg} A$ and $k p s M I I$ was assessed using PCR as described previously [13-18]. The primers sequences are listed in Table 1. PCR was performed using DreamTaq PCR Master Mix (Thermo Fisher Scientific), which contains Taq polymerase, dNTPs, $\mathrm{MgCl}_{2}$ and the appropriate buffer. Each PCR tube contained $25 \mu \mathrm{l}$ reaction mixture composed of $12.5 \mu \mathrm{l}$ of master mix, $1 \mu \mathrm{l}$ of each forward and reverse primer solution (in a final concentration of $200 \mathrm{nM}$ ), $1 \mu \mathrm{l}$ of DNA with concentration of $200 \mathrm{ng} / \mu \mathrm{l}$ and nuclease- free water to complete the volume. The PCR was conducted in a Gene Atlas 322 system (ASTEC). PCR was performed according to the following conditions: initial denaturation at $94{ }^{\circ} \mathrm{C}$ for $5 \mathrm{~min}$, then, 30 cycles of denaturation $\left(94{ }^{\circ} \mathrm{C}, 1 \mathrm{~min}\right)$, annealing (the annealing temperature 
Table 1 Primers sequences and the annealing temperatures used in this study

\begin{tabular}{|c|c|c|c|c|}
\hline $\begin{array}{l}\text { Target } \\
\text { Genes }\end{array}$ & Primers sequences $(5-3)$ & $\begin{array}{l}\text { Annealing } \\
\text { Temperature }\left({ }^{\circ} \mathrm{C}\right)\end{array}$ & $\begin{array}{l}\text { DNA amplicon Size } \\
\text { (bp) }\end{array}$ & Reference \\
\hline epsA & AGCAAGTGGTTATCCAATCG ACCAGACTCACCCATTACAT & 60 & 451 & [12] \\
\hline ompA & CGCTTCTGCTGGTGCTGAAT CGTGCAGTAGCGTTAGGGTA & 58 & 531 & [12] \\
\hline bla $a_{P E R-1}$ & ATGAATGTCATTATAAAAGC AATTTGGGCTTAGGGCAAGAAA & 55 & 927 & [13] \\
\hline bap & $\begin{array}{l}\text { TACTTCCAATCCAATGCTAGGGAGGGTACCAATGCAG TTAT } \\
\text { CCACTTCCAATGATCAGCAACCAAACCGCTAC }\end{array}$ & 55 & 1225 & [14] \\
\hline$b f m S$ & TTGCTCGAACTTCCAATTTATTATAC TTATGCAGGTGCTITITATTGGTC & 60 & 1428 & [14] \\
\hline ptk & GGCTGAGCATCCTGCAATGCGT ACTTCTGGAGAAGGGCCTGCAA & 57 & 597 & [15] \\
\hline pgaB & AAGAAAATGCCTGTGCCGACCA GCGAGACCTGCAAAGGGCTGAT & 57 & 490 & [15] \\
\hline $\mathrm{fimH}$ & TGCAGAACGGATAAGCCGTGG GCAGTCACCTGCCCTCCGGTA & 60 & 870 & [16] \\
\hline $\operatorname{csg} A$ & ACTCTGACTTGACTATTACC GATGCAGTCTGGTCAAC & 50 & 200 & [16] \\
\hline kpsMII & GCGCATTTGCTGATACTGTTG CATCCAGACGATAAGCATGAGCA & 58 & 272 & [16] \\
\hline csuE & ATGCATGTTCTCTGGACTGATGTTGAC CGACTTGTACCGTGACCGTATCTTGATAAG & 60 & 976 & {$[17]$} \\
\hline intl-1 & CAGTGGACATAAGCCTGTTC CCCGAGGCATAGACTGTA & 62 & 160 & [18] \\
\hline int-2 & GTAGCAAACGAGTGACGAAATG CACGGATATGCGACAAAAAGGT & 62 & 788 & [18] \\
\hline intl-3 & GCCTCCGGCAGCGACTTTCAG ACGGATCTGCCAAACCTGACT & 62 & 979 & [18] \\
\hline
\end{tabular}

for each gene are listed in Table 1) for $1 \mathrm{~min}$, extension at $72{ }^{\circ} \mathrm{C}$ for $1 \mathrm{~min}$, followed by a final extension at $72{ }^{\circ} \mathrm{C}$ for 10 min. The amplified DNA was separated by $1 \%$ agarose gel electrophoresis, stained with neutral red (Sigma Aldrich, Germany) and visualized under UV transillumination.

\section{Integron characterization}

Class I, II and III integrons were detected using multiplex PCR as described previously [19]. The primers sequences and PCR conditions are shown in Table 1.

\section{REP-PCR}

The REP like elements in the genomic DNA extracted from $A$. baumannii isolates were amplified with the primer pair REP1 5'-IIIGCGCCGICATCAGGC-3' and REP2 5'-ACGTCTTATCAGGCCTAC-3' as described previously [20]. Amplification was carried out with an initial denaturation at $94{ }^{\circ} \mathrm{C}$ for $10 \mathrm{~min}$, followed by 30 cycles of denaturation $\left(94^{\circ} \mathrm{C}, 1 \mathrm{~min}\right)$, annealing $\left(40{ }^{\circ} \mathrm{C}, 1\right.$ min), extension $\left(72^{\circ} \mathrm{C}, 2 \mathrm{~min}\right)$ and a single final extension at $72{ }^{\circ} \mathrm{C}$ for $16 \mathrm{~min} .20 \mu \mathrm{l}$ of PCR products were subjected to electrophoresis in $2 \%$ agarose gel. Isolates with identical profiles or highly similar profiles (up to 2 bands different) were considered as the same group.

\section{Statistical analysis}

The data were analyzed with SPSS version 17.0 software (SPSS, Inc., Chicago, IL). A chi-square test was used to determine the statistical significance of the data. A $P$ value of $<0.05$ was considered significant.

\section{Results}

\section{Characteristics of isolates}

Out of $100 \mathrm{~A}$. baumannii isolates, 26\% were recovered from sputum, $25 \%$ from wound swabs, $24 \%$ from blood, $15 \%$ from urine and $10 \%$ from secretions collected from chest tube (thoracic catheter).

\section{Antimicrobial susceptibility}

According to our previous study [2], all A. baumannii isolates were resistant to three or more antimicrobial agents and considered as MDR. The highest rate of resistance was detected against ciprofloxacin and imipenem (100\%), followed by piperacillin (99\%) and cefepime/levofloxacin/ ceftazidime (97\%). Furthermore, 32\% of isolates were resistant to all tested antibiotics and 91\% were XDR. The antibiotic resistance patterns of $A$. baumannii isolates are shown in Table 2. The most prevalent pattern was resistance to "ampicillin/sulbactam-ceftazidime-imipenem-gentamicintobramycin-doxycycline-ciprofloxacin-levofloxacin-cotrimoxazole-pipercillin-cefepime" with $32 \%$ frequency.

\section{Biofilm formation assay}

All A. baumannii isolates were able to produce biofilm; 42 isolates produced moderate biofilm and 58 isolates showed strong ability to biofilm formation.

\section{Distribution of biofilm related genes}

All $A$. baumannii isolates carried at least one biofilm related gene. The frequency of these genes is shown in Table 3. The most prevalent gene was csuE (100\%), followed by pgaB (98\%), eps A and ptk (95\%), bfmS (92\%) and $о m p A(81 \%)$. The $\operatorname{csg} A$ and fimH genes were not 
Table 2 Antibiotic resistance patterns of A. baumannii isolates

\begin{tabular}{|c|c|c|}
\hline $\begin{array}{l}\text { No. of antimicrobial } \\
\text { agents }\end{array}$ & Antibiotic resistance patterns & $\begin{array}{l}\text { Percent (\%) of all } \\
\text { isolates }\end{array}$ \\
\hline 5 & Ciprofloxacin-Tobramycin-Gentamicin-Imipenem-Ampicillin/Sulbactam & 2 \\
\hline 7 & $\begin{array}{l}\text { Ceftazidime-Imipenem-Ciprofloxacin-Levofloxacin-Cotrimoxazole-Piperacillin- } \\
\text { Cefepime }\end{array}$ & 2 \\
\hline \multirow[t]{3}{*}{8} & $\begin{array}{l}\text { Ceftazidime-Imipenem-Doxycycline-Ciprofloxacin-Levofloxacin-Cotrimoxazole- } \\
\text { Piperacillin-Cefepime }\end{array}$ & 5 \\
\hline & $\begin{array}{l}\text { Levofloxacin-Ampicillin/Sulbactam-Ceftazidime-Imipenem-Ciprofloxacin- } \\
\text { Cefepime-Piperacillin-Cotrimoxazole }\end{array}$ & 8 \\
\hline & $\begin{array}{l}\text { Ceftazidime-Imipenem-Doxycycline-Ciprofloxacin-Levofloxacin-Cotrimoxazole- } \\
\text { Piperacillin-Cefepime }\end{array}$ & 1 \\
\hline \multirow[t]{3}{*}{9} & $\begin{array}{l}\text { Ampicillin/Sulbactam-Ceftazidime-Imipenem-Doxycycline-Ciprofloxacin-Levofloxacin- } \\
\text { Cefepime-Piperacillin-Cotrimoxazole }\end{array}$ & 20 \\
\hline & $\begin{array}{l}\text { Ampicillin/Sulbactam-Ceftazidim-Imipenem-Gentamycin-Ciprofloxacin-Levofloxacin- } \\
\text { Cotromoxazole-Piperacillin-Cefepime }\end{array}$ & 2 \\
\hline & $\begin{array}{l}\text { Ceftazidime-Imipenem-Gentamicin-Tobramycin-Ciprofloxacin-Levofloxacin- } \\
\text { Cotrimoxazole-Cefepime-Piperacillin }\end{array}$ & 4 \\
\hline \multirow[t]{3}{*}{10} & $\begin{array}{l}\text { Ampicillin/Sulbactam-Ceftazidime-Imipenem-Gentamicin-Tobramycin-Ciprofloxacin- } \\
\text { Levofloxacin-Cotrimoxazole-Piperacillin-Cefepime }\end{array}$ & 20 \\
\hline & $\begin{array}{l}\text { Ampicillin/Sulbactam-Ceftazidime-Imipenem-Gentamicin-Tobramycin-Doxycycline- } \\
\text { Ciprofloxacin-Levofloxacin-Piperacillin-Cefepime }\end{array}$ & 2 \\
\hline & $\begin{array}{l}\text { Ceftazidime-Imipenem-Gentamicin-Tobramycin-Doxycycline-Ciprofloxacin-Levofloxacin- } \\
\text { Cotrimoxazole-Piperacillin-Cefepime }\end{array}$ & 2 \\
\hline 11 & $\begin{array}{l}\text { Ampicillin/Sulbactam-Ceftazidime-Imipenem-Gentamicin-Tobramycin-Doxycycline-Ciprofloxacin- } \\
\text { Levofloxacin-Cotrimoxazole-Pipercillin-Cefepime }\end{array}$ & 32 \\
\hline
\end{tabular}

detected in any A. baumannii isolate. Patterns of biofilm related genes in $A$. baumannii isolates are shown in Table 3. Ninety-eight percent of isolates carried more than 4 biofilm related genes, simultaneously. The most common pattern of simultaneous presence of biofilm related genes was "bfmS-csuE-epsA-bap-kpsMT-ompAptk-pgaB" with 26\% frequency (Table 4). Distribution of biofilm related genes among antibiotic resistant $A$.

Table 3 Frequency of biofilm related genes among $A$.

\begin{tabular}{ll} 
baumannii isolates & No $=(\%)$ of isolates \\
\hline Biofilm related genes & 98 \\
\hline pgaB & 81 \\
ompA & 43 \\
Bap & 92 \\
bfmS & 95 \\
ptk & 95 \\
epsA & 57 \\
kPSMII & 2 \\
bla PER-1 & 0 \\
fimH & 0 \\
CSgA & 0 \\
CSUE & 100 \\
\hline
\end{tabular}

baumannii isolates is shown in Table 5 . The most antibiotic resistant isolates carried several biofilm related genes.

\section{Integron characterization}

The presence of integrons was confirmed in 67 isolates, of which 67 (67\%) and 10 (10\%) cases were identified as class I (intI-1) and class II (intI-2) integrons, respectively. $10 \%$ of isolates harbored both intI-1 and intI-2, simultaneously. However, class I integron was more frequent in comparison with class II $(P<0.05)$. Class III integron was not detected in any $A$. baumannii isolate.

\section{REP-PCR}

The patterns generated with REP-PCR contained several bands, ranging in size from 100 to $4750 \mathrm{bp}$ (Fig. 1). These patterns were classified as 8 types $(\mathrm{A}-\mathrm{H})$ and 21 subtypes. Among 21 subtypes, 14 distinctive REP-PCR clusters and 7 singleton isolates were inferred from the band patterns. The A1 (23\%) and C1 (15\%) clusters were the most prevalent among $A$. baumannii isolates $(P<$ 0.05) (Table 6). According to the REP-PCR assays, $23 \%$ of all isolates had a clonal relatedness. Furthermore, the correlation between REP types (A-H) and biofilm related genes are shown in Table 7. The frequency of biofilm related gene of bap was significantly higher among REP type A $(\mathrm{P}<0.05)$. However, no significant association has 
Table 4 Patterns of biofilm related genes among A. baumannii isolates

\begin{tabular}{|c|c|c|}
\hline No. of biofilm related genes & Patterns of biofilm related genes & Percent (\%) of A. baumannii \\
\hline 4 gene & bfmS-epsA-pgaB-csuE & 2 \\
\hline \multirow[t]{4}{*}{5 gene } & bfmS- csuE-ompA-ptk-pgaB & 5 \\
\hline & bfmS- csuE- epsA - ptk-pgaB & 13 \\
\hline & csuE- epsA - ompA-ptk-pgaB & 3 \\
\hline & csuE- epsA-bap-ompA-ptk & 2 \\
\hline \multirow[t]{4}{*}{6 gene } & bfmS- csuE- epsA-ompA-ptk- pgaB & 8 \\
\hline & csuE- epsA- bap-ompA-ptk-pgaB & 3 \\
\hline & bfmS- csuE- epsA -kpsMT- ompA- pgaB & 3 \\
\hline & bfmS- csuE- epsA- bap- ptk- pgaB & 3 \\
\hline \multirow[t]{5}{*}{7 gene } & csuE- epsA- bap-kpsMT- ompA- ptk-pgaB & 2 \\
\hline & bfmS- csuE - bap -kpsMT- ompA- ptk- pgaB & 2 \\
\hline & bfmS- csuE- epsA -kpsMT- ompA- ptk- pgaB & 21 \\
\hline & bfmS- csuE- epsA- bap - ompA- ptk- pgaB & 3 \\
\hline & bfmS- csuE- epsA- bap -kpsMT- ptk- pgaB & 2 \\
\hline 8 gene & bfmS- csuE- epsA- bap -kpsMT- ompA- ptk- pgaB & 26 \\
\hline 9 gene & bfmS- csuE- epsA-bla $a_{P E R-1}-b a p-k p s M T-o m p A-p t k-p g a B$ & 2 \\
\hline
\end{tabular}

been found between the REP types and other biofilm related genes.

\section{Discussion}

Persistence and survival of MDR A. baumannii in various hospital environments has made it as a major cause of nosocomial infections worldwide [4]. Treatment of infections caused by MDR A. baumannii is complicated in Asian countries such as Turkey, India and Iran [2]. In our study, $100 \%$ of A. baumannii isolates were multidrug resistant. According to previous studies from Iran, the frequency of MDR A. baumannii isolates ranged from 32.7 to 93\% [21]. Furthermore, $32 \%$ of isolates were resistant to all tested antibiotics and 91\% were XDR, which agrees with other investigations conducted in Iran [21, 22]. Similar to our results, 98\% of A. baumannii isolates tested in Saffari et al. study were XDR [22]. It seems that Iran is a hotspot region for the emergence of XDR A. baumannii which is serious problem in healthcare settings. Our results also highlight the significance of XDR in A. baumannii, because all XDR isolates originated from ICU patients. $100 \%$ of isolates were resistant to imipenem and this result is consistent with observations reported from various parts of the world which explain the high risk of failure of carbapenem treatment in A. baumannii infections $[3,5,19,21,22]$. Recently, there are limited options for treatment of carbapenem-resistant $A$. baumannii infections and colistin and tigecycline are considered the last choice to infection control [2]. Previous studies showed that resistance to ampicillinsulbactam in A. baumannii isolates is increasing in ICU patients [23]. Our study also reports this tendency, with a high frequency of ampicillin-sulbactam resistant isolates (87\%), posing another challenge to adequate treatment of A. baumannii infections. The high frequency of antibiotic resistance in our survey is the most probably due to the extensive misuse of antimicrobial agents in our country.

The presence of integrons as a primary source of antimicrobial resistance genes within microbial populations is an important factor in the emergence of MDR isolates [22]. In our study, the high frequency of class I integron (67\%) in comparision with class II was found (10\%), which agrees with other investigations $[19,22]$. We also found a significant association between the presence of class I integron and XDR phenotype $(P<0.01)$. Since class I integrons carrying multiple antibiotic resistance gene cassettes, this association is not unexpected. The first report of class I integron in MDR A. baumannii from northwest Iran showed that $92.5 \%$ of MDR isolates carried class I integron. Significant association between the presence of class I integron and MDR phenotype was reported in their study and other studies [19, 22, 24].

Capability of $A$. baumannii to colonize and form biofilm on biotic and abiotic surfaces is considered as an important factor contributing in chronic and persistence infections [5]. According to our results, all A. baumannii isolates were able to produce biofilm and $58 \%$ of isolates showed strong ability to biofilm formation. Our results are consistent with previous reports which showed that more than $75 \%$ of $A$. baumannii isolates form biofilms [5, 6]. Previous studies have reported a positive relationship between biofilm formation and antibiotic resistance in A. baumannii isolates [7, 25]. In our study, all strong biofilm forming A. baumannii isolates were XDR. We 


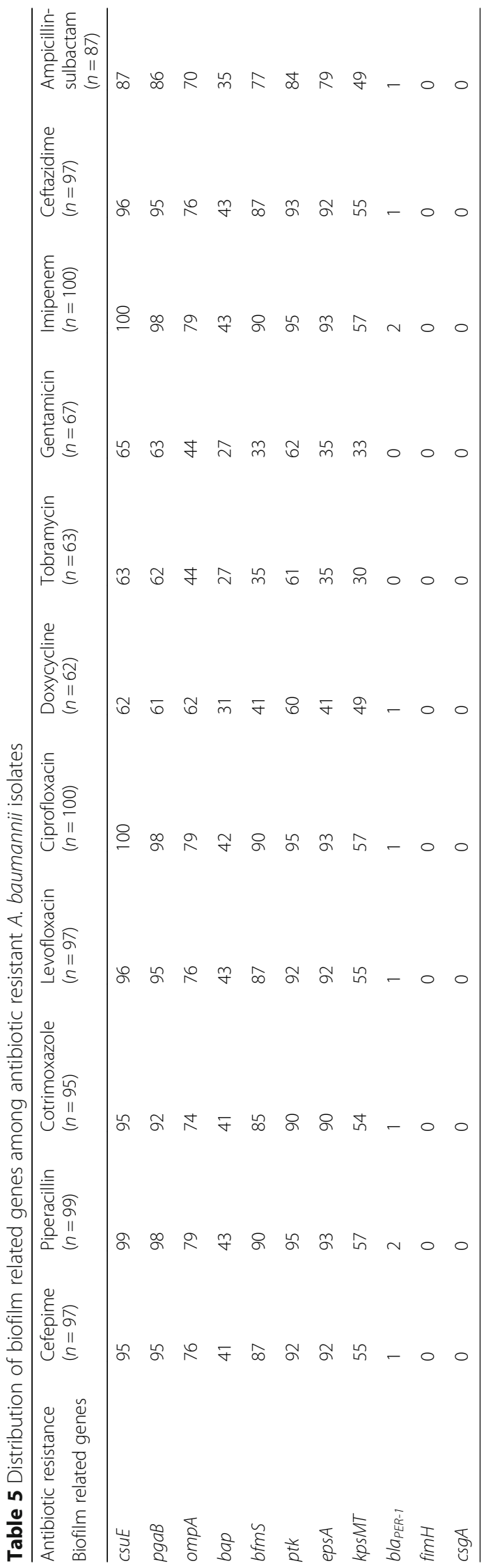




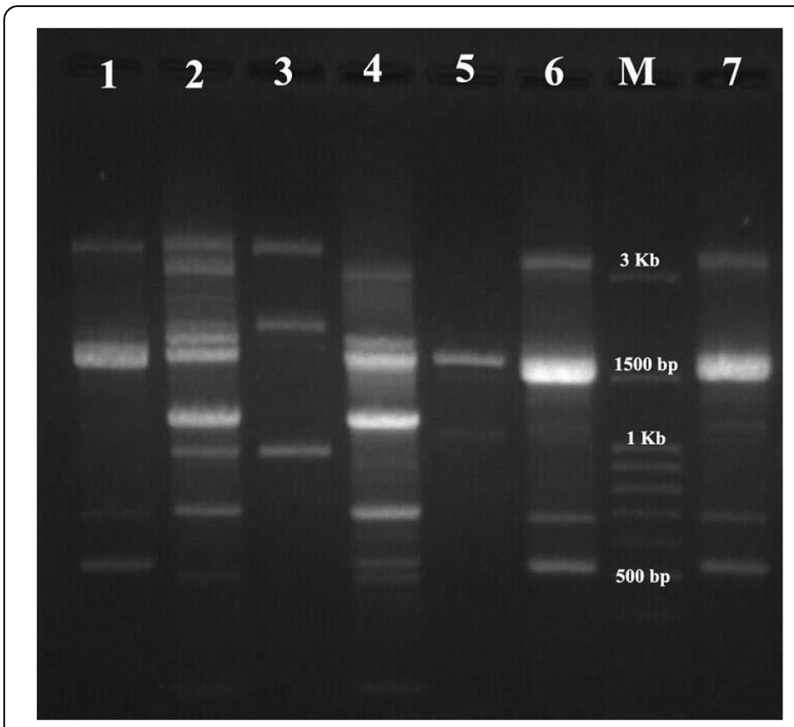

Fig. 1 REP- PCR patterns identified among A. baumannii isolates examined in this study. Lane 1-7: clinical isolates, Lane M: DNA marker (100 bp) found significant correlation between strong biofilm formation and XDR phenotype $(P<0.01)$. Several reports have demonstrated that biofilm related genes of $A$. baumannii including csuE, ompA, bap, epsA, bfmS were responsible for biofilm development and antibiotic resistance [4-6]. According to our results, the most prevalent gene was $c s u E$ (100\%), followed by pgaB (98\%), epsA and ptk (95\%), bfmS (92\%) and ompA (81\%). Previous studies were also reported the high frequency of $c s u E$ in $A$. baumannii isolates, so that $\operatorname{csuE}$ was detected in 100 and $93.8 \%$ of isolates in Ghasemi et al. and Youn Sung studies, respectively $[4,6]$. The OmpA of $A$. baumannii is probably essential for the attachment to human epithelial cells, development of biofilms and antimicrobial resistance [5]. There was high frequency of ompA in our study $(81 \%)$. Similar results were reported from Thailand and Korea with 84.4 and $68.8 \%$ ompA positive isolates, respectively $[5,6]$. All strong biofilm forming A. baumannii isolates were carried csuE, pgaB, ptk, epsA, bfmS and ompA genes, simultaneously. However, these biofilm related genes were also detected in some moderate biofilm forming A. baumannii isolates. According to high frequency of biofilm related genes in A. baumannii isolates, the strong biofilm formation was expected. In agreement with previous studies, bla $_{\text {PER-1 }}$ was less common in our study and only 2 isolates harbored this gene $[4,6]$. However, recent report

Table 6 REP-PCR patterns among A. baumannii isolates

\begin{tabular}{|c|c|c|c|}
\hline REP Types & Subtypes & Size of REP-PCR products (bp) & Total isolates number (\%) \\
\hline \multirow[t]{2}{*}{ A (3 bands) (24\%) } & $\mathrm{A} 1$ & $800,1000,3000$ & 23 \\
\hline & $\mathrm{A} 2$ & $700,1500,2500$ & 1 \\
\hline \multirow[t]{2}{*}{ B (4 bands) (4\%) } & $\mathrm{B} 1$ & $700,850,1000,1600$ & 3 \\
\hline & B2 & $900,1400,1800,3000$ & 1 \\
\hline \multirow[t]{5}{*}{ C (5 bands) (27\%) } & $\mathrm{C} 1$ & $900,950,1000,1500,2000$ & 15 \\
\hline & $\mathrm{C} 2$ & $800,1000,1500,2500,3000$ & 9 \\
\hline & $\mathrm{C} 3$ & $700,1600,2000,3000,4000$ & 1 \\
\hline & $\mathrm{C} 4$ & $550,700,800,1000,3100$ & 1 \\
\hline & C5 & $750,1000,1900,2900,3000$ & 1 \\
\hline \multirow[t]{4}{*}{ D (6 bands) (14\%) } & D1 & $800,1300,2200,2500,3000,3400$ & 4 \\
\hline & D2 & $700,1000,1900,2300,3000,3300$ & 4 \\
\hline & D3 & $650,900,1900,2700,3000,4750$ & 3 \\
\hline & D4 & $900,1000,1200,1500,2000,3000$ & 3 \\
\hline \multirow[t]{3}{*}{ E (7 bands) (16\%) } & E1 & $750,800,1000,1300,1500,2500,3000$ & 6 \\
\hline & E2 & $650,850,900,1300,2000,2800,3000$ & 9 \\
\hline & E3 & $250,450,900,1400,2100,2700,3000$ & 1 \\
\hline \multirow[t]{2}{*}{ F (8 bands) (7\%) } & $\mathrm{F} 1$ & $600,700,900,1200,1600,2000,2800,3200$ & 3 \\
\hline & $\mathrm{F} 2$ & $100,300,400,800,1000,1500,2800,3000$ & 4 \\
\hline \multirow[t]{2}{*}{ G (9 bands) (7\%) } & G1 & $300,500,800,900,1600,2300,2800,3200,4300$ & 4 \\
\hline & G2 & $100,300,500,650,700,900,1100,1700,3000$ & 3 \\
\hline H (10 bands) (1\%) & $\mathrm{H}$ & $100,250,500,700,800,1000,1500,2000,2700,3000$ & 1 \\
\hline
\end{tabular}


Table 7 Correlation between REP types and biofilm related genes among A. baumannii isolates

\begin{tabular}{|c|c|c|c|c|c|c|c|c|c|c|c|}
\hline $\begin{array}{l}\text { REP } \\
\text { Types }\end{array}$ & $\begin{array}{l}\text { pgaB } \\
(n=98)\end{array}$ & $\begin{array}{l}\text { ompA } \\
(n=81)\end{array}$ & $\begin{array}{l}\text { Bap } \\
(n=43)\end{array}$ & $\begin{array}{l}\text { bfmS } \\
(n=92)\end{array}$ & $\begin{array}{l}\text { Ptk } \\
(n=95)\end{array}$ & $\begin{array}{l}\text { eps } A \\
(n=95)\end{array}$ & $\begin{array}{l}\text { kpsMII } \\
(n=57)\end{array}$ & $\begin{array}{l}b^{\prime} a_{P E R-1} \\
(n=2)\end{array}$ & $\begin{array}{l}\text { fimH } \\
(n=0)\end{array}$ & $\begin{array}{l}\operatorname{csg} A \\
(n=0)\end{array}$ & $\begin{array}{l}\text { csuE } \\
(n=100)\end{array}$ \\
\hline $\begin{array}{l}\text { A (3 bands) } \\
(24 \%)\end{array}$ & 24 & 23 & $19^{*}$ & 22 & 24 & 23 & 18 & 2 & 0 & 0 & 24 \\
\hline $\begin{array}{l}\text { B (4 bands) } \\
(4 \%)\end{array}$ & 2 & 3 & 1 & 4 & 4 & 4 & 2 & 0 & 0 & 0 & 4 \\
\hline $\begin{array}{l}\text { C (5 bands) } \\
(27 \%)\end{array}$ & 27 & 22 & 8 & 25 & 25 & 26 & 17 & 0 & 0 & 0 & 27 \\
\hline $\begin{array}{l}\text { D (6 bands) } \\
(14 \%)\end{array}$ & 14 & 10 & 4 & 12 & 14 & 13 & 6 & 0 & 0 & 0 & 14 \\
\hline $\begin{array}{l}\text { E (7 bands) } \\
(16 \%)\end{array}$ & 16 & 12 & 6 & 16 & 15 & 15 & 8 & 0 & 0 & 0 & 16 \\
\hline $\begin{array}{l}\text { F (8 bands) } \\
(7 \%)\end{array}$ & 7 & 6 & 2 & 5 & 7 & 6 & 3 & 0 & 0 & 0 & 7 \\
\hline $\begin{array}{l}\text { G (9 bands) } \\
(7 \%)\end{array}$ & 7 & 5 & 2 & 7 & 6 & 7 & 2 & 0 & 0 & 0 & 7 \\
\hline $\begin{array}{l}\text { H (10 bands) } \\
(1 \%)\end{array}$ & 1 & 0 & 1 & 1 & 0 & 1 & 1 & 0 & 0 & 0 & 1 \\
\hline
\end{tabular}

${ }^{*} P$ value of $<0.05$ was considered as significant

from Thailand found bla $a_{\mathrm{PER}-1}$ in $30.2 \%$ of $A$. baumannii isolates [7].

The molecular typing of A. baumannii is an important tool for determination of genetic and epidemiological relatedness. Repetitive extragenic palindromic sequence based polymerase chain reaction (REP-PCR) is a suitable method for comparison of genetic profiles of A. baumannii [26]. According to Grisold et al. [27] and Pasanen et al. [26] studies, the discriminatory power of REP-PCR was found to be sufficiently high and corresponded reasonably well with PFGE. We used REP-PCR technique for A. baumannii typing and according to the results, the size of REP-PCR products ranged from 100 to $4750 \mathrm{bp}$. These patterns were classified as 8 types (A-H) and 21 subtypes. Among 21 subtypes, 14 distinctive REP-PCR clusters and 7 singleton isolates were inferred from the band patterns The A1 (23\%) and $\mathrm{C} 1(15 \%)$ clusters were the most prevalent among $A$. baumannii isolates $(P<0.05)$. According to the REP-PCR patterns, $23 \%$ of all isolates had a clonal relatedness. Similar to our results, Meshkat et al. was reported that REP-PCR tying of clinical $A$. baumannii isolates generate 10 distinctive clusters (named A to J) and eight singleton isolates. According to their results, up to $94 \%$ of all the strains were included in nine distinct clusters and only $6 \%$ of them had common roots [28]. In the previous study from Iran, genotypic comparison by REP-PCR revealed that carbapenem resistant isolates belonged to six clones and all clones were spread in the ICUs. Clone A was dominant (30.9\%) and clone F had the lowest prevalence (1\%) [29]. In our study, the frequency of biofilm related gene of bap was significantly higher among REP type A $(P<0.05)$. However, no significant association has been found between the REP types and other biofilm related genes. In study conducted by Dahdouh et al., international clones I and III (IC I, III) was negatively associated with $\alpha$-hemolysis and strong biofilm formation $(p<0.05)$. But, international clone II and those harboring blaOXA-24-like were positively associated with $\alpha$-hemolysis, production of strong biofilms, and siderophore production [1].

\section{Conclusion}

Our study revealed the high frequency of biofilm forming XDR $A$. baumannii in ICU patients, with a high prevalence of biofilm related genes of $c s u E$ and $p g a B$. It seems that the appropriate surveillance and control measures are essential to prevent the emergence and transmission of XDR A. baumannii in our country.

\section{Abbreviations}

Bap: Biofilm Associated Protein; Csu: Chaperon- usher Pilus; EPS: Extracellular Exopolysaccharide; HLAR: High Level Aminoglycoside Resistance;

MDR: Multidrug Resistant; OmpA: Outer Membrane Protein A;

VRE: Vancomycin Resistant Enterococci; XDR: Extensively Drug Resistance

\section{Acknowledgements}

The authors would like to acknowledge Mehdi Kashefiyeh for his support in sampling.

\section{Authors' contributions}

$\mathrm{FH}$ and $\mathrm{HZ}$ involved in the project development, data management and manuscript writing. FV, RS and ES participated in project development, data analysis and manuscript preparation. All authors have read and approved the final version of manuscript.

\section{Funding}

This work as an MSc thesis in Medical Microbiology was supported by the Zanjan University of Medical Sciences (A-12-535-20).

\section{Availability of data and materials}

The datasets will not be available on a publically available website, but it may be possible to provide access to anonymized data. Anyone who wants to request the data can contact with Fakhri Haghi, corresponding author. 


\section{Ethics approval and consent to participate}

Ethical clearance was obtained from the Ethics Committee of Zanjan University of Medical Sceinces before conducting the study (IR.ZUMS.REC.1396.278). All participants provided written informed consent to participate in the studies.

\section{Consent for publication}

Not applicable.

\section{Competing interests}

The authors declare that they have no competing interests.

\section{Author details}

'Department of Microbiology, School of Medicine, Zanjan University of Medical Sciences, Zanjan, Iran. 'Department of Microbiology, Zanjan Branch, Islamic Azad University, Zanjan, Iran.

Received: 8 April 2019 Accepted: 10 July 2019

Published online: 17 July 2019

\section{References}

1. Dahdouh E, Gómez-Gil R, Pacho S, Mingorance J, Daoud Z, Suárez M. Clonality, virulence determinants, and profiles of resistance of clinical Acinetobacter baumannii isolates obtained from a Spanish hospital. PLoS One. 2017;27:1-18

2. Shirmohammadlou N, Zeighami H, Haghi F, Kashefieh M. Resistance pattern and distribution of carbapenemase and antiseptic resistance genes among multidrug-resistant Acinetobacter baumannii isolated from intensive care unit patients. J Med Microbiol. 2018;67(10):1467-73.

3. Safari M, Saidijam M, Bahador A, Jafari R, Alikhani MY. High prevalence of multidrug resistance and metallo-beta-lactamase (MbetaL) producing Acinetobacter baumannii isolated from patients in ICU wards, Hamadan, Iran. J Res Health Sci. 2013;13(2):162-7.

4. Ghasemi E, Ghalavand Z, Goudarzi H, Yeganeh F, Hashemi A, Dabiri H, Mirsamadi ES, Foroumand M. Phenotypic and Genotypic Investigation of Biofilm Formation in Clinical and Environmental Isolates of Acinetobacter baumannii. Arch Clin Infect Dis. 2018;13(4):e12914.

5. Thummeepak R, Kongthai P, Leungtongkam U, Sitthisak S. Distribution of virulence genes involved in biofilm formation in multi-drug resistant Acinetobacter baumannii clinical isolates. Int Microbiol. 2016;19(2):121-9.

6. Youn Sung J. Molecular Characterization and Antimicrobial Susceptibility of Biofilm-forming Acinetobacter baumannii Clinical Isolates from Daejeon, Korea, vol. 50; 2018.

7. Qi L, Li H, Zhang C, Liang B, Li J, Wang L, Du X, Liu X, Qiu S, Song H. Relationship between antibiotic resistance, biofilm formation, and biofilmspecific resistance in Acinetobacter baumannii. Front Microbiol. 2016;7:483.

8. Chapartegui-Gonzalez I, Lazaro-Diez M, Bravo Z, Navas J, Icardo JM, Ramos-Vivas J. Acinetobacter baumannii maintains its virulence after long-time starvation. PLoS One. 2018;13(8):e0201961.

9. Amala Reena A, Subramaniyan A, Kanungo R. Biofilm formation as a virulence factor of Acinetobacter baumannii: An emerging pathogen in critical care units. J Curr Res Sci Med. 2017;3(2):74-78.

10. Laboratory Standards Institute antimicrobial susceptibility testing standards M02-A12, M07-A10, and M11-A8. 2017.

11. Magiorakos AP, Srinivasan A, Carey RB, Carmeli Y, Falagas ME, Giske CG, Harbarth S, Hindler JF, Kahlmeter G, Olsson-Liljequist B, et al. Multidrugresistant, extensively drug-resistant and pandrug-resistant bacteria: an international expert proposal for interim standard definitions for acquired resistance. Clin Microbiol Infect. 2012;18(3):268-81.

12. Toledo-Arana A, Valle J, Solano C, Arrizubieta MJ, Cucarella C, Lamata M, Amorena B, Leiva J, Penadés JR, Lasa I. The enterococcal surface protein, Esp, is involved in enterococcus faecalis biofilm formation. Appl Environ Microbiol. 2001;67(10):4538-45

13. Tayabali AF, Nguyen KC, Shwed PS, Crosthwait J, Coleman G, Seligy VL. Comparison of the virulence potential of Acinetobacter strains from clinical and environmental sources. PLoS One. 2012:7(5):e37024.

14. Lee HW, Koh YM, Kim J, Lee JC, Lee YC, Seol SY, Cho DT, Kim J. Capacity of multidrug-resistant clinical isolates of Acinetobacter baumannii to form biofilm and adhere to epithelial cell surfaces. Clin Microbiol Infect. 2008;14(1):49-54.
15. Liou ML, Soo PC, Ling SR, Kuo HY, Tang CY, Chang KC. The sensor kinase BfmS mediates virulence in Acinetobacter baumannii. J Microbiol Immunol Infect Wei mian yu gan ran za zhi. 2014;47(4):275-81.

16. Bahador A, Bazargani A, Taheri M, Hashemizadeh Z, Khaledi A, Rostami H, Esmaeili D. Clonal Lineages and Virulence Factors among Acinetobacter baumannii Isolated from Southwest of Iran, vol. 7; 2013.

17. Braun $G$, Vidotto MC. Evaluation of adherence, hemagglutination, and presence of genes codifying for virulence factors of Acinetobacter baumannii causing urinary tract infection. Mem Inst Oswaldo Cruz. 2004;99(8):839-44.

18. Turton JF, Gabriel SN, Valderrey C, Kaufmann ME, Pitt TL. Use of sequencebased typing and multiplex PCR to identify clonal lineages of outbreak strains of Acinetobacter baumannii. Clin Microbiol Infect. 2007;13(8):807-15.

19. Najar Peerayeh S, Karmostaji A. Molecular identification of resistance determinants, Integrons and genetic relatedness of extensively drug resistant Acinetobacter baumannii isolated from hospitals in Tehran, Iran. Jundishapur J Microbiol. 2015;8(7):e27021.

20. Bou G, Cervero G, Dominguez MA, Quereda C, Martinez-Beltran J. PCRbased DNA fingerprinting (REP-PCR, AP-PCR) and pulsed-field gel electrophoresis characterization of a nosocomial outbreak caused by imipenem- and meropenem-resistant Acinetobacter baumannii. Clin Microbiol Infect. 2000;6(12):635-43.

21. Vahdani P, Yaghoubi T, Aminzadeh Z. Hospital acquired antibiotic-resistant acinetobacter baumannii infections in a 400-bed hospital in Tehran, Iran. Int J Prev Med. 2011;2(3):127-30.

22. Saffari F, Monsen T, Karmostaji A, Bahadori Azimabad F, Widerström M. Significant spread of extensively drug-resistant Acinetobacter baumannii genotypes of clonal complex 92 among intensive care unit patients in a university hospital in southern Iran, vol. 66; 2017.

23. Castilho SRA, Godoy CSM, Guilarde AO, Cardoso JL, Andre MCP, JunqueiraKipnis AP, Kipnis A. Acinetobacter baumannii strains isolated from patient in intensive care units in Goiania, Brazil: molecular and drug susceptibility profiles. PLoS One. 2017;12(5):e0176790.

24. Peymani A, Farajnia S, Nahaei MR, Sohrabi N, Abbasi L, Ansarin K, Azhari F. Prevalence of class 1 integron among multidrug-resistant Acinetobacter baumannii in Tabriz, northwest of Iran. Pol J Microbiol. 2012;61(1):57-60.

25. Babapour E, Haddadi A, Mirnejad R, Angaji S-A, Amirmozafari N. Biofilm formation in clinical isolates of nosocomial Acinetobacter baumannii and its relationship with multidrug resistance. Asian Pac J Trop Biomed. 2016;6(6):528-33.

26. Pasanen T, Koskela S, Mero S, Tarkka E, Tissari P, Vaara M, Kirveskari J. Rapid molecular characterization of Acinetobacter baumannii clones with rep-PCR and evaluation of carbapenemase genes by new multiplex PCR in Hospital District of Helsinki and Uusimaa. PLoS One. 2014;9(1):e85854.

27. Grisold AJ, Zarfel G, Strenger V, Feierl G, Leitner E, Masoud L, Hoenigl M, Raggam RB, Dosch V, Marth E. Use of automated repetitive-sequence-based PCR for rapid laboratory confirmation of nosocomial outbreaks. J Infect. 2010;60(1):44-51.

28. Meshkat Z, Salimizand H, Amini Y, Khakshoor M, Mansouri D, Farsiani H, Ghazvini K, Najafi A. Molecular characterization and genetic relatedness of clinically Acinetobacter baumanii isolates conferring increased resistance to the first and second generations of tetracyclines in Iran. Ann Clin Microbiol Antimicrob. 2017;16(1):51.

29. Shoja S, Moosavian M, Rostami S, Abbasi F, Tabatabaiefar MA, Peymani A. Characterization of Oxacillinase and Metallo- $\beta$-Lactamas genes and molecular typing of clinical isolates of Acinetobacter baumannii in Ahvaz, south-west of Iran. Jundishapur J Microbiol. 2016;9(5):e32388.

\section{Publisher's Note}

Springer Nature remains neutral with regard to jurisdictional claims in published maps and institutional affiliations. 